\title{
Precondicionamiento farmacológico con sildenafilo del riñón con isquemia normotérmica
}

\author{
Lledó García E, Rodríguez Martínez D*, Cabello Benavente R, Dulín Elena**, García Bordas J***, \\ Fernández Álvarez E***, Hernández Fernández C, del Cañizo López JF*. \\ Servicios de Urología, *Medicina y Cirugía Experimental, **Bioquimica y ***Anatomía Patológica. Hospital \\ General Universitario Gregorio Marañón. Madrid.
}

Actas Urol Esp. 2008;32(1):67-74

\section{RESUMEN}

PRECONDICIONAMIENTO FARMACOLÓGICO CON SILDENAFILO DEL RIÑÓN CON ISQUEMIA NORMOTÉRMICA Objetivos: Evaluar el posible efecto pre-condicionador de sildenafilo en riñones sometidos a isquemia normotérmica, perfusión hipotérmica y posterior autotrasplante.

Material y Métodos: Estudiamos 6 grupos de órganos autotrasplantados: control sin isquemia y autotrasplante inmediato (grupo A); control con $45 \mathrm{~min}$ de isquemia y autotrasplante inmediato (grupo B); isquemia + perfusión del órgano en bomba y autotrasplante (grupo C); isquemia + conservación mediante hipotermia simple en solución UW y autotrasplante (grupo D); $100 \mathrm{mg}$ vo sildenafilo preoperatorio+isquemia+perfusión en bomba y autotrasplante (grupo D); $100 \mathrm{mg}$ vo sildenafilo preoperatorio+ isquemia+ autotrasplante inmediato (grupo E). Evaluamos durante los 60 minutos de reperfusión postrasplante los valores medios de flujo vascular renal (FVR), resistencia vascular renal (RVR), presión arterial sistémica (PAS) y concentración de óxido nítrico en vena del injerto renal (ON). Realizamos estudio histológico mediante microscopia electrónica y convencional en todos los casos. Resultados: Obtuvimos unos valores medios de flujo vascular renal (FVR) mayores, resistencia vascular renal menor (RVR) y concentraciones de óxido nítrico en vena del injerto (ON) mayores en los primeros 60 min de reperfusión renal postrasplante en los grupos E y F frente a A,B,C y D (Tabla 1, Fig. 1). Desde el punto de vista histológico, los órganos sometidos a trasplante sin isquemia, perfundidos tras la isquemia o tratados preoperatoriamente con sildenafilo mostraron integridad túbulo-endotelial en los estudios microscópicos.

Conclusiones: Mostramos, por primera vez en la literatura específica, un efecto beneficioso de sildenafilo en los parámetros obtenidos en la reperfusión postrasplante en los riñones sometidos a isquemia normotérmica durante un periodo crítico.

Palabras clave: Sildenafilo. Trasplante renal. Isquemia normotérmica.

\section{ABSTRACT}

PHARMACOLOGICAL PRECONDITIONING WITH SILDENAFIL OF WARM ISCHEMIC KIDNEYS

Objectives. To evaluate the preconditioning effect of sildenafil administered preoperatively in kidneys subjected to a period of warm ischemia (WI), hypothermic perfusion (HP) or cold storage (CS) and finally, autotransplant (AT).

Material and method. We studied 6 groups of autotransplanted kidneys: no-WI-inmediate AT (Group A); 45 min of WI + immediate AT (Group B); 45 min of WI +60 min of HP + autotransplant (Group C); 45 min of WI + 60 min of CS + autotransplant (Group D); $100 \mathrm{mg}$ of oral sildenafil preoperatively + $45 \mathrm{~min}$ of WI + autotransplant (Group E); $100 \mathrm{mg}$ of oral sildenafil preoperatively $+45 \mathrm{~min}$ of WI+60 min of HP + autotransplant (Group F). Belzer solution was used for HP; UW-Viaspan for CS. Inmediately after the autotransplant (reperfusion period), we recorded in real time for 60 min the values of Renal vascular Flow (RVF) and Renal Vascular Resistance (RVR). Nitric Oxide levels in the cava and renal graft vein were recorded every $15 \mathrm{~min}$ during the $60 \mathrm{~min}$ of the reperfusion-study period. Conventional \& Electronic microscopy were completed after the process.

Results. We obtained significant higher values of RVF and lower values of RVR in sildenafil groups ( $E$ and F) in comparison to the other groups (A-D) (Table 1). NO levels were also significantly higher in groups $\mathrm{E}$ and $\mathrm{F}$ (Fig. 1). Groups A, B, E and F showed integrity of tubule and endothelium in comparison to groups C and D in the microscopic study.

Conclusions. We showed a beneficious effect of sildenafil in inmediate post-transplant reperfusion hemodynamic and biochemical parameters of kidneys subjected to a critical period of warm-ischemia.

Keywords: Sildenafile. Renal Transplantation. Warm ischemia. 
$\mathrm{L}$ a isquemia normotérmica puede producir distintos niveles de lesión endotelial en los órganos susceptibles de trasplante ${ }^{1}$. La disfunción del endotelio vascular durante el proceso de reperfusión (lesión por isquemia-reperfusión) ${ }^{2}$. Sildenafilo puede intensificar la acción del GMP cíclico (GMPc), aumentar indirectamente la producción de óxido nítrico $(\mathrm{ON})$ y atenuar la disfunción endotelial. El mecanismo de acción de este fármaco implica la inhibición de la enzima fosfodiesterasa tipo 5 (PDE5), lo que resultaría en un aumento de la concentración de GMPc y relajación del músculo liso ${ }^{3}$. Estudios inmunohistoquímicos han demostrado la presencia de PDE5 en músculo liso vascular y bronquial, así como en plaquetas ${ }^{4}$.

No está actualmente aún claro cuál es el mejor sistema de preservación de los riñones que provienen de donantes a corazón parado (NHBD). La administración de óxido nítrico en estos donantes durante la isquemia caliente y la posterior reperfusión del órgano trasplantado ha mostrado en diseños experimentales de trasplante pulmonar una disminución del edema del injerto ${ }^{2,3}$. Por otro lado, en los últimos años se ha producido un creciente interés en investigar el papel de los inhibidores de la fosfodiesterasa tipo 5 (IPDE5) en la protección frente a la isquemiareperfusión en modelos animales. Kukreja ${ }^{5}$ ha mostrado de forma convincente que el fármaco puede inducir un efecto protector pre-condicionador en el miocardio isquémico. Algunos trabajos han mostrado la capacidad de los fármacos inmunosupresores inhibidores de la calcineurina (ciclosporina, tacrolimus) en inducir una vasoconstricción renal dosis dependiente, reversible, que afecta a la arteriola aferente; según estos mismos trabajos, sildenafilo podría aumentar el filtrado glomerular en pacientes trasplantados, presumiblemente revirtiendo este efecto $^{6,7}$. Ningún estudio ha sido, sin embargo, publicado sobre la posible acción de los IPDE5 en riñones con isquemia normotérmica y posterior implante.

En este trabajo experimental evaluamos los efectos hemodinámicos, bioquímicos e histológicos de sildenafilo en los órganos sometidos a isquemia normotérmica y posterior trasplante durante el crítico periodo de la reperfusión postdesclampaje.

\section{MATERIAL Y MÉTODOS. DISEÑO EXPERIMENTAL}

Utilizamos 30 cerdos de laboratorio (raza large white: peso $40-45 \mathrm{~kg}$ ). Se siguió estrictamente la Normativa Internacional de cuidado de animales de experimentación durante todo el estudio. Los animales fueron anestesiados con $15 \mathrm{mg} / \mathrm{kg}$ de ketamina y 0,02 $\mathrm{mg} / \mathrm{kg}$ de atropina: la ventilación mecánica se llevó a cabo mediante un respirador (Boyle Modular, BOC, Surrey, U.K.), en modo de control de volumen. La anestesia se mantuvo mediante una mezcla de halotano al $2 \%$, fentanilo y bromuro de pancuronio.

Los animales se distribuyeron de la siguiente forma:

- Grupo A: Control sin isquemia-trasplante inmediato (modelo donante vivo, $n=5$ ).

- Grupo B: Control con $45 \mathrm{~min}$ de isquemia normotérmica y trasplante inmediato $(n=5)$.

- Grupo C: Isquemia normotérmica de 45 minutos, 60 minutos de perfusión hipotérmica del riñón aislado en bomba de perfusión, trasplante $(\mathrm{n}=5)$.

- Grupo D: Isquemia normotérmica de 45 minutos, 60 minutos de conservación hipotérmica del riñón mediante inmersión en solución UWViaspán a $4^{\circ} \mathrm{C}$, trasplante $(\mathrm{n}=5)$.

- Grupo E: Administración de 100 mg de sildenafilo vía oral 1 hora antes de la cirugía. Isquemia normotérmica de 45 minutos, 60 minutos de perfusión hipotérmica del riñón aislado en bomba de perfusión, trasplante $(n=5)$.

- Grupo F: Administración de 100 mg de sildenafilo vía oral 1 hora antes de la cirugía. Isquemia normotérmica de 45 minutos, trasplante $(n=5)$.

El procedimiento de isquemia normotérmica fue uniforme: 45 minutos de isquemia mediante clampaje arterial y venoso. Posteriormente, se completó la nefrectomía simple derecha en todos los casos. Todos los órganos fueron sometidos a lavado por gravedad, transarterial, con solución de Universidad de Wisconsin-Viaspan, antes de distribuirlos al grupo correspondiente. La perfusión hipotérmica se realizó en un dispositivo de bombeo pulsátil controlado por vacío durante $60 \mathrm{~min}$ a $4^{\circ} \mathrm{C}$ con solución de Belzer. Antes de completar el autotrasplante a aorta y cava, se realizó la nefrectomía izquierda. La preservación mediante hipotermis 
simple (Grupo C) se llevó a cabo con solución de Wisconsin-Viaspan. Tras el desclampaje se registró en tiempo real durante 60 minutos (periodo de isquemia reperfusión agudo) el flujo vascular renal (FVR), presión arterial sistémica (PAS), resistencia vascular renal (RVR) mediante transductores de presión aórtico y flujo en arteria renal específicos. El procedimiento completo fue monitorizado y registrado mediante un sistema informatizado.

Se tomaron muestras de sangre de vena renal y vena cava para determinación de óxido nítrico a $0^{\prime}, 15^{\prime}, 30^{\prime}, 45^{\circ}, 60^{\prime} \mathrm{min}$ tras el desclampaje. El ON producido fue determinado con un ensayo espectrofotométrico, mediante la medición de los productos acumulados de degradación estables, nitritos y nitratos. Para monitorizar ambos llevamos a cabo la conversión enzimática de nitratos en nitritos mediante la nitrato reductasa, seguida por la cuantificación de nitritos mediante el reactante de Griess. Previamente a iniciar el ensayo, las muestras que contenian altos niveles proteicos fueron filtradas, para evitar un precipitado que interfiriese con la medición. Se preparó una curva estándar en un rango de $0-100 \mu \mathrm{M}$ de ON.

Se realizaron estudio microscópicos con microscopia convencional y electrónica en todos los casos tras el procedimiento. La tinción de hematoxilina-eosina se usó en la microscopia convencional; la técnica de Reynolds (tinción ultraestructural de acetato de uranilo y citrato plúmbico) para la microscopia electrónica (JEOLJEM 100 SXR).

En cuanto al diseño experimental de nuestro trabajo, debemos referir lo siguiente. Se evaluaron 4 factores: isquemia normotérmica, perfusión hipotérmica, hipotermia simple y administración de sildenafilo. Los niveles para cada factor fueron 2 (sí/no). Las variables analizadas, 4: flujo vascular renal, resistencia vascular renal, presión arterial sistémica y concentración de óxido nítrico. Finalmente, los tratamientos planteados, seis (los referidos).

\section{RESULTADOS (Tabla 1)}

Todos los grupos de animales fueron homogéneos en cuanto al peso y cifras de presión arterial sistémica media prequirúrgica. No observamos tampoco diferencias significativas en relación a la presión arterial media entre los grupos $\mathrm{E}$ y $\mathrm{F}$ (animales que habian recibido medicación oral preoperatoria) respecto a los demás grupos en todos los tiempos de medición postrasplante. Sin embargo, el grupo D (isquemia + conservación hipotérmica pretrasplante) presentó unos valores de presión arterial media significativamente menores frente al resto de grupos y para todos los tiempos de medición post-desclampaje (Fig. 1).

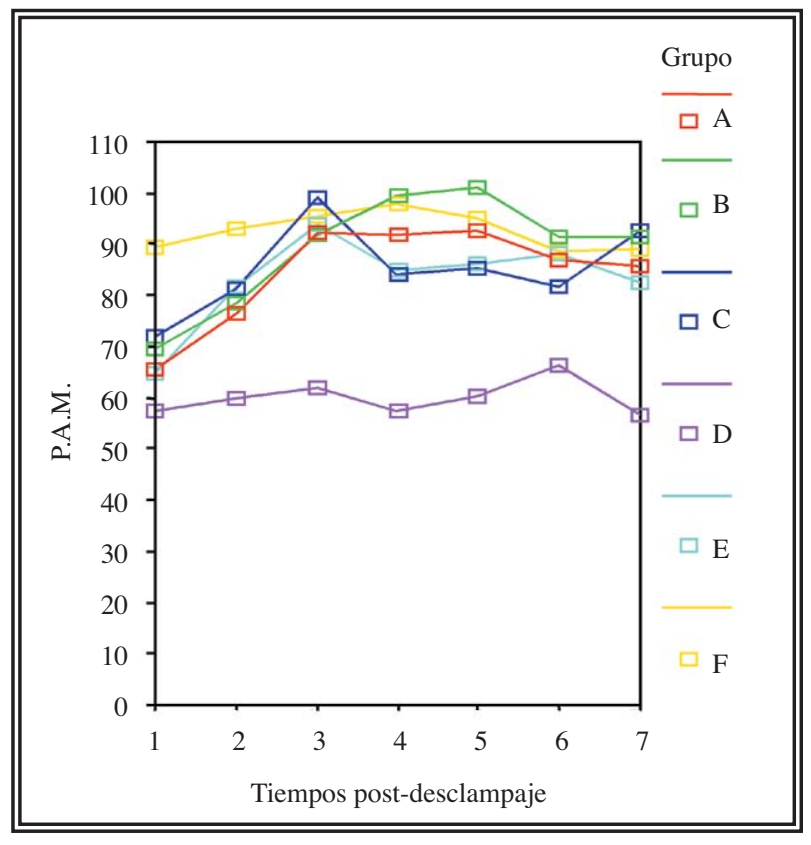

FIGURA 1

Tabla 1. Valores medios de presión arterial sistémica, flujo vascular renal, resistencia vascular renal y concentración de Oxido nítrico según el grupo de estudio (en rojo: valores en grupos con administración de sildenafilo pre-isquemia)

\begin{tabular}{lcccccc}
\hline Variables/Grupos & A & B & C & D & E & F \\
\hline Presion arterial sistémica (Mmhg) & 84,3 & 88,9 & 85,15 & 61,01 & 83,04 & 91,04 \\
Flujo vascular renal (Ml/Min/100 g) & 75,10 & 62,91 & 52,55 & 73,03 & $\mathbf{1 3 3 , 9 2}$ & $\mathbf{1 7 2 , 9 8}$ \\
Resistencia vascular renal (Mmhg/Ml/Min) & 1,89 & 3,06 & 2,66 & 1,22 & $\mathbf{0 , 9 1}$ & $\mathbf{0 , 5 8}$ \\
Oxido nítrico $(\mu \mathrm{M})$ & 15,98 & 7,28 & 21,84 & 10,73 & $\mathbf{3 1 . 4 5}$ & $\mathbf{3 8 , 9}$ \\
\hline
\end{tabular}


En cuanto al FVR medido en el injerto tras el desclampaje, pudimos diferenciar durante todo el periodo de registro dos grupos diferenciados. Por un lado, los grupos A, B, C, D (no sildenafilo preoperatorio) frente a los grupos $\mathrm{E}$ y $\mathrm{F}$, con valores de flujo en incremento progresivo, pero con valores absolutos significativamente más altos. Dentro del primer bloque de riñones, el grupo A (no isquemia-trasplante inmediato) mostró más FVR frente a B, C y D; no obstante, el FVR fue claramente inferior que en los grupo E y F. A partir del minuto 20 post-desclampaje, los órganos del grupo C (isquemia-perfusión en bomba-trasplante), mostraron valores absolutos e incrementos de FVR menores (Fig. 2).

Dentro de los grupos E y F (sildenafilo precirugia), los valores mayores de FVR se obtuvieron en el grupo F (isquemia, no preservación, trasplante inmediato). Este grupo mostró valores de FVR significativamente mayores frente a todos los grupos comparados y en todos los tiempos de medición. Parecía, a tenor de estos resultados, que el factor más determinante fue la administración de sildenafilo preoperatorio.

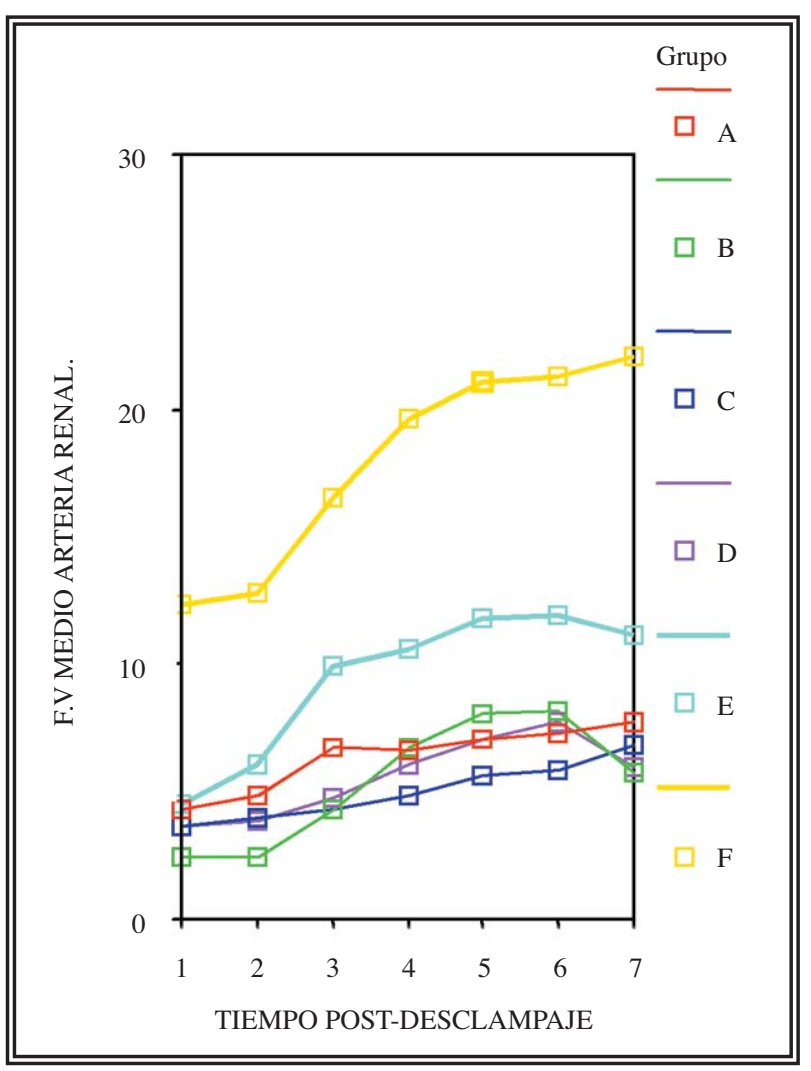

FIGURA 2
En cuanto a la evolución de los valores de resistencia vascular renal (dependientes de la presión y el flujo vascular de la arteria renal), los grupos E y $\mathrm{F}$ mostraron varias características diferenciales: unos valores iniciales de RVR postdesclampaje más bajos, una progresión con pendiente descendente durante todo el proceso, unos valores medios para todos los tiempos de medición inferiores en comparación al resto de grupos. Además, entre los grupo $\mathrm{E}$ y F, los riñones de los animales que habian sido medicados preoperatoriamente sin perfusión posterior del órgano aislado mostraron los valores significativamente más bajos de RVR al final del proceso de monitorización (Fig. 3).

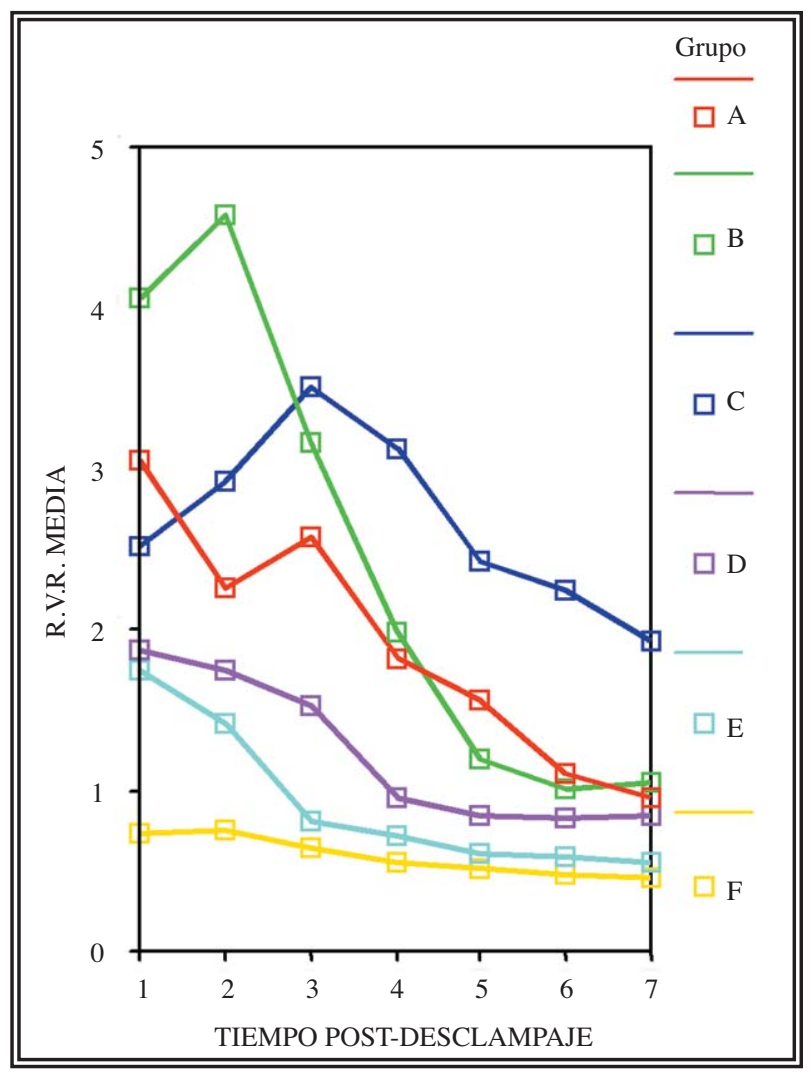

FIGURA 3

Finalmente, las determinaciones de óxido nítrico en sangre venosa mostraron hallazgos concretos e interesantes:

1. Las concentraciones de O.N. en los grupos E y F (pre-tratados con sildenafilo) fueron mayores durante todo el proceso de reperfusión y se mantuvieron homogéneas y sin diferencias significativas de una manera más estable que el resto de los grupos (Fig. 4). 


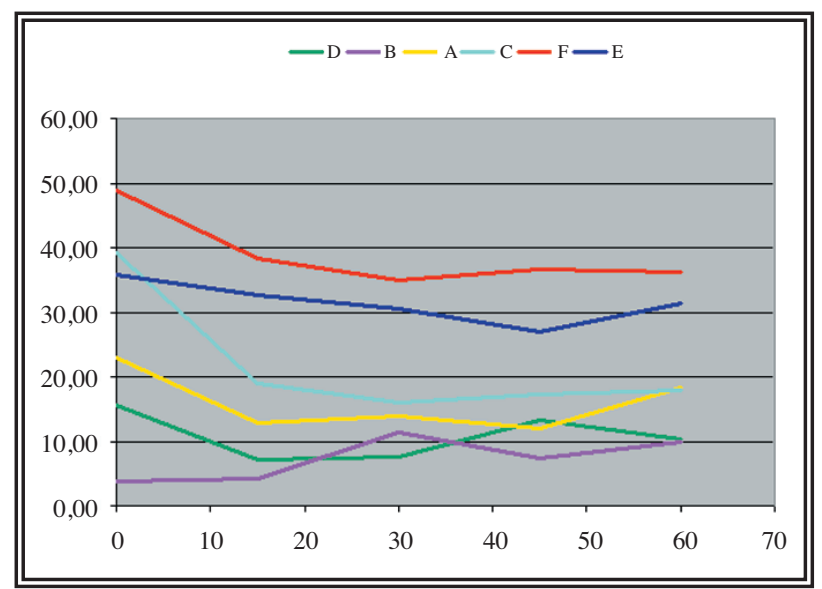

FIGURA 4. Concentraciones medias de óxido nitrico en los diferentes grupos de estudio.

2. Al evaluar si hubo diferencias en la concentración de O.N. vena renal-vena cava entre animales con/sin administración preoperatorio de sildenafilo pudimos ver que, efectivamente, los animales que habian sido pre-medicados mostraban concentraciones sistémicas uniformemente mayores de O.N. que los animales sin pre-tratamiento (Fig. 5).

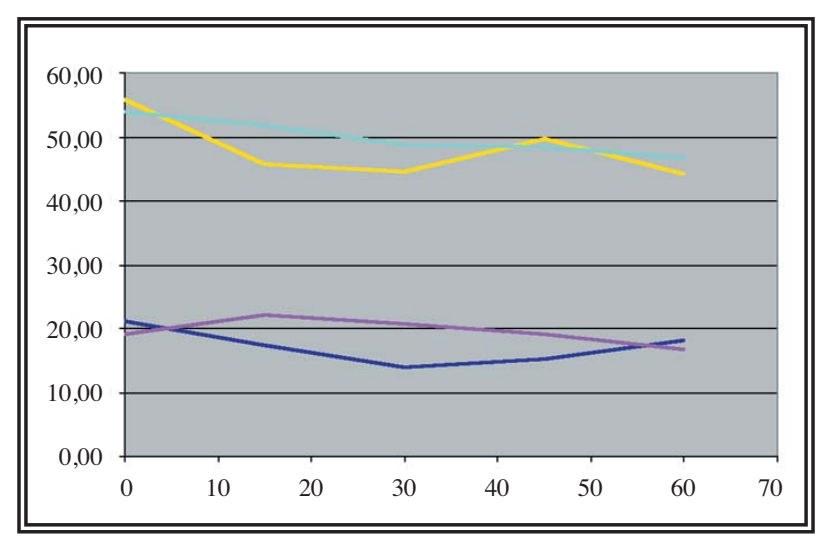

FIGURA 5. Perfil de concentraciones medias de óxido nitrico en vena renal y vena cava según administración (curvas superiores, Verde-amarilla) vs no administración (inferiores, azul-rosa) de sildenafilo preoperatorio, sin diferencias de concentración entre cava y vena renal.

En cuanto a los resultados histológicos, pudimos diferenciar tres grupos principales:

1. Riñones sin isquemia normotérmica o con isquemia normotérmica y perfusión pretrasplante (Grupos A y C): mostraron un patrón de integridad estructural túbulo-glomerulal, discreto edema intersticial, conservación endotelial (Fig. 6).
2. Riñones con isquemia normotérmica y trasplante directo o con isquemia normotérmica y preservación por inmersión hipotérmica simple (Grupos B y D): mostraron un patrón de alteración de la estructura túbulo-glomerular y endotelial (fibrina intraluminal) (Fig. 7).

3. Riñones con sildenafilo -isquemia normotérmica- con/sin perfusión en bomba pretrasplante (Grupos E y F). Mostraron una esplendida conservación de la estructura mitocondrial y endotelial en la microscopia electrónica (Fig. 8).

\section{DISCUSIÓN}

El éxito en los resultados obtenidos en las últimas décadas en el trasplante de órganos sólidos ha producido un aumento en la necesidad de donantes de órganos. El llamado donante a corazón parado es una fuente de órganos de eficacia comprobada $^{8}$. Aunque la isquemia caliente puede, potencialmente, producir importantes lesiones en el injerto, varios grupos de trabajo han mostrado la eficacia de la conservación de estos riñones mediante perfusión hipotérmica en máquina ${ }^{9}$. Nuestro grupo ha presentado trabajos desde hace años con un sistema de perfusión pulsátil regulado por vació y controlado mediante un sistema informático, diseñado por nosotros mismos ${ }^{10}$.

Sea el que sea el sistema de preservación para estos riñones, uno de los efectos de la isquemia normotérmica en el órgano es un incremento en la resistencia vascular, probablemente inducido por la pérdida de capacidad del endotelio vascular renal para producir óxido nítrico y/o, como posibilidad alternativa, un desequilibrio en la producción de endotelina-1/óxido nítrico, lo que es esencial para la función vascular que regula la perfusión del órgano ${ }^{11}$. Valores mayores de resistencia vascular se han relacionado con porcentajes más altos de disfunción inicial del injerto y una supervivencia más baja del mismo ${ }^{8}$.

Sildenafilo, un fármaco inhibidor de la fosfodiesterasa tipo 5 , posee un doble mecanismo potencial de relajación del músculo liso vascular: un mecanismo indirecto, dependiente de óxido nítrico-GMP cíclico y una vía directa posiblemente mediante inhibición de la entrada de calcio iónico a través de los canales de calcio ${ }^{12}$. Es posible que la acción vasodilatadora de sildenafilo pudiese liberar mediadores endógenos precondicionadores tales como la adenosina o la bradiquinina de las células endoteliales, lo cual desen- 

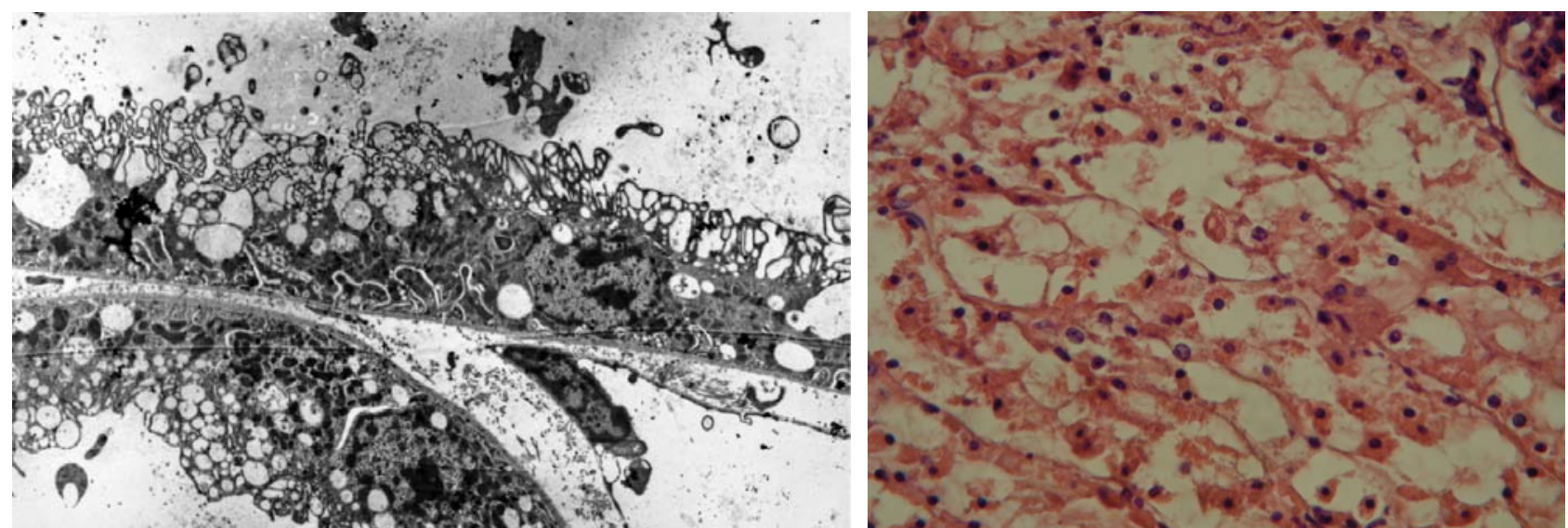

FIGURA 6. Conservación de la estructura túbulo-endotelial en órganos $\sin$ IN y con IN+perfusión en bomba (micro electrónica-micro convencional.
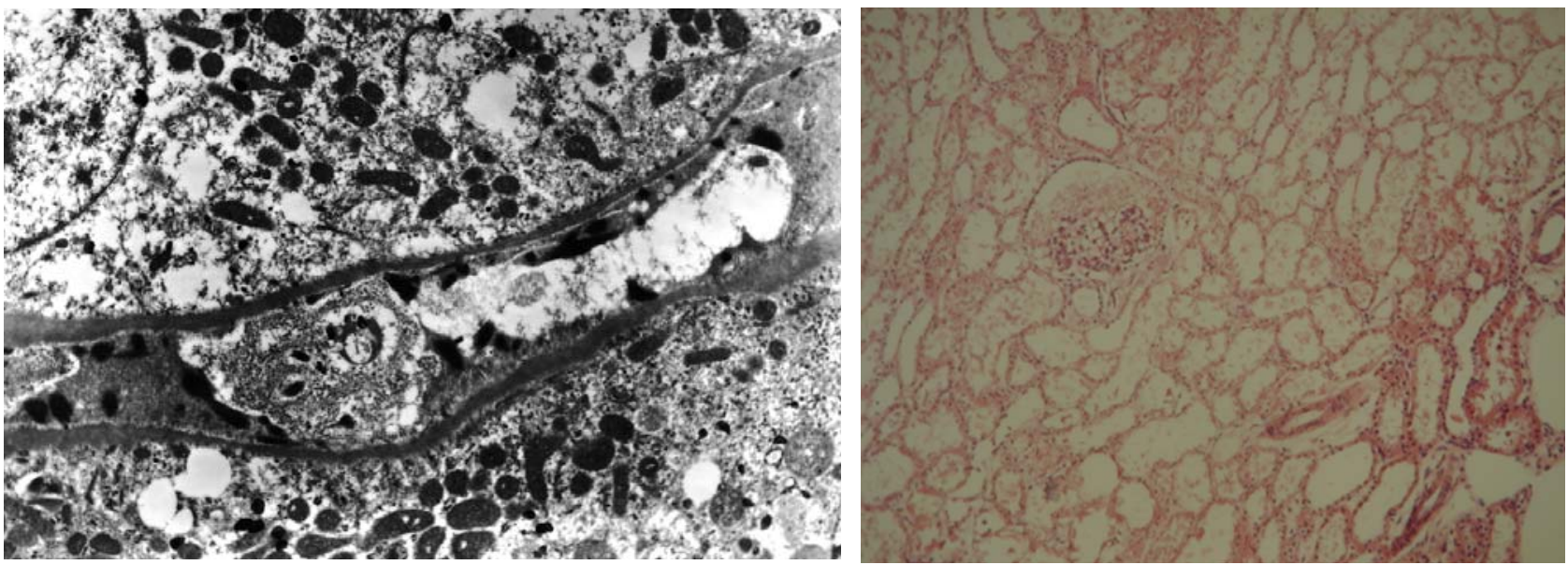

FIGURA 7. Riñones con IN e hipotermia simple o IN sin preservación pre-trasplante: lesiones endoteliales, fibrina intralumnal (micro. Electrónica) - edema tubular, retracción glomerular (micro. Convencional).

cadenará un proceso - efecto cascada (a través de la acción de quinasas) que resultaría en la fosforilación de óxido nítrico sintetasa y la liberación de óxido nítrico ${ }^{5}$. La sintesis de óxido nítrico es catalizada por 3 isoformas de O.N. sintetasa, a saber, neuronal (nNOS), inducible (iNOS) y endotelial (eNOS). Ha sido demostrado que el O.N. derivado de iNOS es, en modelos experimentales, el mediador primario del efecto protector de sildenafilo en tejido miocárdico isquémico. La expresión de ARN mensajero-iNOS determinado con RT-PCR tras el tratamiento con sildenafilo fue, en estos modelos experimentales, máxima a las 2 horas de la administración del fármaco ${ }^{5}$.

Este fármaco ha sido utilizado en clínica, fundamentalmente, en la disfunción eréctil. Sin embargo, se están introduciendo novedosas indicaciones de uso que muestran el extraordinario potencial de los inhibidores de la PDE5. Estas nuevas utilizaciones terapéuticas surgen al hilo

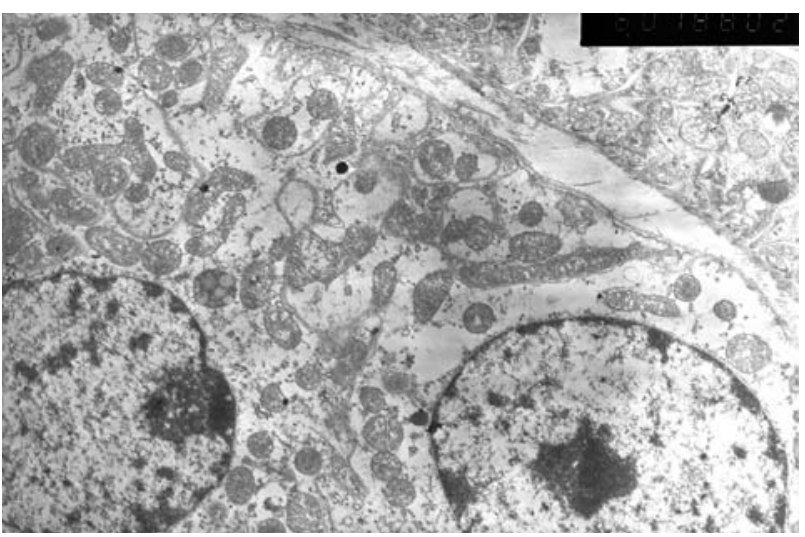

FIGURA 8. Riñones con sildenafilo - IN con/sin perfusión pre-trasplante: integridad endotelial-mitocondrial (micro. Electrónica).

del descubrimiento de que fosfodiesterasa tipo 5 se expresa en diversos tejidos, como la vascularización arterial, la venosa, el músculo esquelético, las plaquetas y el músculo visceral y traqueo-bronquial ${ }^{13}$. 
Sildenafilo ha demostrado mejorar la función endotelial en pacientes con trasplante cardiaco e hipertensión arterial ${ }^{14}$. Este fármaco ha demostrado también ser eficaz en el tratamiento de niños con hipertensión pulmonar primaria ${ }^{15}$. Adicionalmente, se ha publicado recientemente referencias experimentales de un efecto beneficioso de la administración de óxido nítrico a donantes en parada cardíaca durante la isquemia caliente, lo que podría reducir la lesión por isquemia-reperfusión y el extravasado capilar, mejorando los resultados del trasplante de pulmón ${ }^{4}$. No han sido publicadas referencias en la literatura respecto al posible efecto positivo hemodinámica de sildenafilo en trasplantes de riñones con isquemia normotérmica.

Nuestro modelo de autotrasplante intenta excluir el factor inmunológico, que constituiria un sesgo fundamental para la interpretación de los resultados. De esta manera evitamos la interferencia producida por variables no controladas. Nuestro modelo experimental evalúa, por un lado, el papel de sildenafilo como fármaco precondicionador (mediante su administración previamente a la isquemia). Además, el modelo usado evalúa a sildenafilo como fármaco protector de la función endotelial y de sus posibles consecuencias microvasculares, pues el autotrasplante se realiza durante la fase teórica de niveles plasmáticos óptimos. La administración del fármaco 60 minutos antes de iniciar la intervención se ha basado en modelos experimentales de isquemia miocárdica a través de los cuales se ha podido conocer que, tras la administración del inhibidor de PDE5, los niveles máximos de iNOS se consiguen a las 2 horas $^{5}$. En otro orden de cosas, la dosis utilizada $(1,5 \mathrm{mg} / \mathrm{kg})$, en comparación a la dispensada en otros modelos experimentales $(0,7 \mathrm{mg} / \mathrm{kg})$ de peso $^{5}$, intenta evitar los problemas relacionados con la vía de administración (oral Versus intravenosa) para conseguir niveles plasmáticos igualmente eficaces.

Aunque en numerosos trabajos parece que la perfusión en máquina es un método preferible frente a la hipotermia simple en la preservación de riñones con isquemia normotérmica (NHBD), tanto en el plano clínico como en el experimental, no son numerosos los trabajos que intenten crear una alternativa coadyuvante a estos sistemas convencionales de preservación. El modelo de isquemia caliente renal es un planteamiento de lesión endotelial crítica. La respuesta, por tanto, de ese endo- telio al trasplante del órgano será consecuencia, si se quiere conseguir un buen funcionamiento del injerto, de la eficacia de las maniobras de conservación utilizadas. El donante vivo supondría el planteamiento ideal (ausencia de isquemia caliente prolongada, trasplante directo), frente a otro modelo antagónico (isquemia normotérmica, no maniobras de preservación, trasplante).

La medición de la resistencia vascular renal está directamente relacionada con la presión de perfusión y el flujo vascular de la arteria renal. Este parámetro ha sido planteado en diversos trabajos como un criterio con valor pronóstico funcional del injerto. El flujo vascular de la arteria renal es uno de los dos criterios que, junto con la determinación de la alfa-glutation-S-transferasa, ha demostrado cierta evidencia científica en cuanto al carácter pronóstico funcional del injerto ${ }^{16}$. La RVR es un valor resultante que refleja el estado de adaptación vascular a la reperfusión, en un órgano como el riñón que tiene la característica de autorregular el flujo de perfusión mediante la contracción de la musculatura lisa vascular. Esta capacidad dependería, entre otros factores, de la conservación/pérdida de la producción de moléculas vasoactivas por el endotelio renal. La isquemia normotérmica puede, por definición, lesionar el endotelio microvascular, un elemento altamente lábil. Es en ese punto donde las maniobras de preservación deben demostrar su eficacia, que quedará en evidencia especialmente en la respuesta del órgano a la situación más crítica que sufre un injerto tras su extracción e isquemia: la reperfusión tras el trasplante. Nosotros obtuvimos unos valores significativamente mayores de FVR y menores de RVR, junto con concentraciones más altas de O.N. en los dos grupos de riñones en los que habiamos administrado sildenafilo. La mejor respuesta vascular era evidente. Nos llamó la atención que la perfusión en máquina no tuvo un efecto adicional al combinarla con la administración del fármaco: incluso, el grupo F mostró valores de FVR mayores al grupo $\mathrm{E}$.

Las concentraciones de ON detectados en los grupos E y F resultaron ser, además de mayores, más estables a lo largo del proceso de registro. Este hecho podria ser interpretado como una mejor adaptación funcional del endotelio tras la agresión isquémica normotérmica, en respuesta, probablemente a sildenafilo. La consecuencia serían los menores valores de RVR en estos grupos en comparación al resto de grupos de estudio. 
El modelo que presentamos en este trabajo unifica en un solo caso a donante y receptor, pues el fármaco es administrado antes de la isquemia. Nuestro objetivo próximo será diferenciar los efectos del fármaco cuando éste sea administrado por vía intravenosa tras la isquemia, antes del implante (efecto puro en receptor) y diseñar un modelo de supervivencia.

Es importante remarcar que no observamos diferencias en los valores de presión arterial sistémica entre los animales con/sin sildenafilo preoperatorio. Esto podría interpretarse como que sildenafilo produce un efecto positivo, actuando específicamente en el endotelio vascular alterado por la isquemia y mejorando su función, sin producir efectos colaterales sistémicos.

El análisis de los resultados histológicos obtenidos traduce el efecto de la agresión aplicada a los órganos en cada uno de los grupos. En los riñones sin isquemia previa (Grupo A, modelo de donante vivo), las alteraciones microscópicas fueron menores, con poco edema y sin alteraciones túbulo-endoteliales. Sin embargo, la concentración sérica de O.N. no fue la mayor así como el perfil hemodinámico, siendo aceptable, no fue el mejor de los grupos. Con unos perfiles similares encontramos los órganos con isquemia normotérmica y perfusión en máquina pre-trasplante (Grupo C). Este tiempo de preservación resultó positivo, atenuando las lesiones si lo comparamos con los grupos B o D. Los grupos E y F mostraron una histología similar al grupo A, y tuvieron, sin embargo, un perfil hemodinámico y unos niveles de O.N. claramente superiores. Vemos, pues, que la correlación histológico-hemodinámica/bioquímica no fue completa. Es, probablemente el factor funcional-endotelial el que marca la diferencia entre los grupos de estudio. Podríamos suponer que la administración de sildenafilo produce un efecto de mejoría funcional en el endotelio que resulta determinante para interpretar los resultados en su conjunto.

La perfusión pulsátil hipotérmica en bomba ha sido considerada en diversos trabajos como el método de elección para los riñones de donantes a corazón parado, al conseguir mejores valores de FVR y RVR ${ }^{17}$. Sin embargo, la administración de fármacos que protegen la función endotelial como los inhibidores de la PDE5 a donante/receptor puede mejorar o incluso bastar para paliar o atenuar los efectos de la agresión isquémica.

\section{CONCLUSIONES}

Nuestro resultados sugieren ${ }^{18,19}$, un posible efecto protector de sildenafilo en la respuesta de injertos renales con isquemia caliente crítica, sin efectos secundarios sistémicos.

\section{REFERENCIAS}

1. Mi H, Carter V, Gupta A, Asher J, Shenton BK, Stamp S, et al. Anti-Vimentin antibody detection in recipients of heart-beating and non-heart-beating donor kidneys. Transpl Proc. 2005;37(8):3269-3271.

2. Egan TM, Hoffmann SC, Mayura Sevala, Sadoff JD, Schlidt SA. Nitroglycerin reperfusion reduces ischemia-reperfusion injury in non-heart-beating donor lungs. J Heart Lung Transplant 2006;25(1):110-119

3. Cirino G, Fusco F, Imbimbo C, Mirone V. Pharmacology of erectile dysfunction in man. Pharmacology Therapeutics. 2006;111(2):400-423.

4. Takashima S, Koukoulis G, Inokawa H, Sevala M, Egan TM. J Thorac Cardiovasc Surg. 2006;132(1):132-139.

5. Kukreja RC, Salloum F, Das A, Ockaili R, Yin C, Bremer YA, et al. Pharmacological preconditioning with sildenafil: Basic mechanisms and clinical implications. Vascular Pharmacology. 2005;42(5-6):219-232.

6. Christ B, Brockmeier D, Hauck EW, Kamali-Ernst S. Investigations on interaction between tacrolimus and sildenafil in kidney transplanted patients with erectile dysfunction. Int J Clin Pharmacol Ther. 2004;42(3):149-156.

7. Rostaing L, Tran-Van T, Ader JL. Increased glomerular filtration rate in kidney-transplant recipients who take sildenafil. N Engl J Med. 2000;342(22): 1679-1680.

8. Booster MH, Kootstra G. The Non-Heart Beating Donor, a new source for kidney transplantation. Clinical and Experimental Studies. Universitaire pers Maastricht Ed. Maastricht (The Netherlands), 1995.

9. St Peter SD, Imber CJ, Friend PJ. Liver and Kidney preservation by perfusion. Lancet. 2002;359(9306):604-613.

10. Lledó-Garcia E, Hernández-Fernández C, Díez-Cordero JM, Garcia-Barreno P, del Cañizo-López JF. Hydrodynamic and biochemical effects of isolated hypothermic renal perfusion depending on the pump model and perfusion solution. Transplantation Procceedings 2003; 35(5):1661-1663.

11. Jeong GY, Chung KY, Lee WJ, Kim YS, Sung SH. The effect of a nitric oxide donor on endogenous endothelin-1 expression in renal ischemia-reperfusion injury. Transpl Proc. 2004;36(7):1943-1945.

12. Lau LC, Adaikan PG. Mechanisms of direct relaxant effect of sildenafil, tadalafil and vardenafil on corpus cavernosum. Eur J Pharmacol. 2006;541(3): 184-190.

13. Raja SG, Nayak SH. Sildenafil: emerging cardiovascular indications. Ann Thorac Surg. 2004;78(4):1496-1506.

14. Schofield RS, Edwards DG, Schuler BT, Estrada J, Aranda JM, Pauly DF, et al. Vascular effects of sildenafil in hypertensive cardiac transplant recipients. Am J Hypertens. 2003;16(10):874-877.

15. Fraisse A, Habib G. Traitement de l'hypertension artérielle pulmonaire de l'enfant. Archives de Pédiatrie. 2004;11(8): 945-950.

16. Wight J, Chilcott J, Holmes M, Brewer N. The clinical and cost-effectiveness of pulsatile machine perfusion versus cold storage of kidneys for transplantation retrieved from heart-beating and non-heart-beating donors. Health Technol Assess. 2003;7(25):1-94.

17. Hansen TN, D`Alessandro A, Southard JH. Reduced renal vascular injury following warm ischemia and preservation by hypothermic machine perfusion. Transpl Proc. 1997;29 (8):3577-3579.

18. Lledó E, Rodriguez D, Cabello R, Moncada I, Hurtado J, Dulin E, Hernández C, Del cañizo JF. Sildenafil improves immediate post-transplant parameters in warm ischemic kidney transplants: experimental study. Transplantation Procceedings. Accepted for publication (2007). IN PRESS.

19. Lledó E, Moncada I, Rodriguez D, Cabello R, Dulin E, Del Canizo JF, Hernandez C Preconditioning effect of sildenafil in warm-ischemic kidney transplants: experimental results. American Urological Congress-2007 Anaheim. Abstract 90372

Correspondencia autor: Dr. E. Lledó García

Servicio de Urologia. Hospital General Univ. Gregorio Marañon Doctor Esquerdo, 46 - 28007 Madrid. Tel.: 915868000

E-mail autor: ENLLGA@terra.es

Información artículo: Original 